\title{
Capacidade Máxima de Abertura Bucal em Crianças de 3 a 12 Anos de Idade
}

\author{
Eleutério Araújo Martins* \\ Fernando Araújo** \\ Rejane Nunes*** \\ Marcia Michel $* * *$ \\ Carlos Feldens***** \\ Jacques Öhr*****
}

\begin{abstract}
RESUMO
Cinqüenta e seis pacientes foram examinados na Clínica de Odontopediatria da Faculdade de Odontologia da UFRGS com a finalidade de determinar um limite normal de sua capacidade máxima de abertura bucal. Eles foram divididos em três grupos de idade, 3 a 5, 6 a 8, e 9 a 12 anos. 0 método utilizado é bastante simples e pode ser realizado em um curto espaço de tempo. Os resultados obtidos indicam que o método pode ser incluído em um exame de rotina e em Odontopediat ria

e pode servir como um alerta no que se refere à desordens do sistema estomatognático em crianças.
\end{abstract}

\section{SUMMARY}

Fifty six patients were examined at the Pedodontic Clinic, School of Dentistry, UFRGS, in order to determine a normal limit of their maximum opening mouth capacity. They were divided in three groups of ages, 3 to 5,6 to 8 , eno 9 to 12. The method used is quite simple an easy to be done in a short time. The

results obtained indicate that it can be included in a routine pedodontic examination and be used as an alert as far as prevention of disorders of the stomatognathic system is concerned.

\section{DESCRITORES}

Odontopediatria; Oclusão Dentária; Fisiologia.

*Professor Titular. Disciplina de Oclusão, FO-UFRGS.

**Prof. Adjunto. Disciplina de Odontopediatria, FO-UFRGS.

***Profa. Auxilliar da Disciplina de Oclusão, FO-UFRGS.

$* * *, * * * *, * * * * *$ Internos da Disciplina de Odontopediatria, FO-UFRGS. 
Um dos sintomas que fazem parte da Síndrome de Dor e Disfunção dos músculos e ATM (SDDM e ATM) é a diminuição da capacidade de movimentação mandibular.

Diversos trabalhos estabeleceram parâmetr os para os limites entre função normal e disfunção do SEG.

Atualmente é aceita como uma si tuação de normalidade e capacidade de aber tura máxima de $45 \mathrm{~mm}$ para mulheres e $50 \mathrm{~mm}$ para homens, adultos.

\section{OBJETIVO}

Face às limitações de informações tanto na li teratura corrente como em amostragens de nossa população, decidimos com o presente trabalho buscar a determinação de valores médios, correspondentes à máxima abertura bucal em pacientes jovens, em faixas etárias determinadas, com a finalidade de possibilitar o estabelecimento de um parâmetro de normalidade que possa ser incorporado à rotina do exame clínico odontopediátrico.

\section{MATERIAL E MÉTODO}

Foi elaborada inicialmente uma ficha suscinta onde constavam os dados essenciais do paciente, estado de saúde oral e abertura em milímetros (máxima) de cada um.

\section{AMOSTRA}

A amostra consistiu de 56 pacientes que procuraram atendimento na Clínica de Odontopediatria do curso de graduação da UFRGS.

Esses pacientes foram divididos em três grupos experimentais de acordo com o desenvolvimento da dentição. A saber:

1. Dentição Decídua: $N=21-p a-$ cientes de 3 a 5 anos de idade.

2. Dentição Mista: $\mathbf{N}=23$ - pacientes de 6 a 8 anos de idade (dentição Decídua mais 1.:s molares per- manentes, mais incisivos permanentes).

3. Dentição Permanente jovem: $\mathrm{N}=$ 12 - pacientes de 9 a 12 anos de idade.

\section{METODOLOGIA}

O grau de abertura bucal foi medido com auxílio de um paquímetro sensível ao décimo de milímetro. Ao número obtido foi somado o eventual e variável sobrepasse vertical dos incisivos ou diminuído o grau de afastamento entre os incisivos ainda não completamente erupcionados fisiologicamente ou patologicamente (mordida aberta anterior).

A tomada da distância foi feita entre os bordos incisais dos: $11 / 41$ ou $21 / 31$ ou $51 / 81$ ou $61 / 71$.

\section{RESULTADOS}

Os resultados obtidos foram analisados estatisticamente para a obtenção de valores médios para as três faixas etárias alvo da observação.

$\begin{array}{rccc} & n & \bar{x} & s \\ 3-5 \text { anos } & 21 & 42,23 & 4,95 \\ \text { 6-8 anos } & 23 & 46,34 & 5,38 \\ 9-12 \text { anos } & 12 & 48,41 & 6,01\end{array}$

Com a média do universo estatístico $=\mu$

$$
\begin{aligned}
& \text { 3- } 5 \text { anos }-39,98 \text { e } 44,48 \mathrm{~mm} \\
& 6-8 \text { anos }-44,02 \text { e } 48,66 \mathrm{~mm} \\
& 9-12 \text { anos }-44,61 \text { e } 52,21 \mathrm{~mm} \\
& p<0,05
\end{aligned}
$$

\section{AVALIAÇÃO dOS RESULTADOS}

Os dados encontrados em nosso trabalho e apresentados pelos três grupos não mostram grande variabilidade dentro dos grupos. Contudo, quando comparados grupo com grupo de 3-5 anos difere dos outros dois.

Da análise dos dados pode-se afirmar que a média do universo estatístico, em $95 \%$ dos casos estará entre os valores:

\section{3- 5 anos de idade: 39,98 e $44,48 \mathrm{~mm}(p<0,05)$ \\ 6- 8 anos de idade: 44,02 e $48,66 \mathrm{~mm}(\mathrm{p}<0,05)$ \\ 9-12 anos de idade: $44,61 \mathrm{e}$ $52,21 \mathrm{~mm}(\mathrm{p}<0,05)$}

\section{DISCUSSÃO}

Os resultados encontrados, nos permitem o estabelecimento de números significativos de situações de aparente normalidade clínica. Esse estabelecimento está reforçado pelos resultados encontrados por outros pesquisadores em faixas etárias semelhante e/ou próximas. Nevakari (1960) encontrou em 50 meninos e 50 meninas entre 6-8 anos de idade a média de $46,00 \mathrm{~mm}$ (5). Sheppard (1965) em 14 crianças 3-5 anos de idade o valor de $42,40 \mathrm{~mm}$ e $21 \mathrm{crian}$ ças entre 6-10 anos de idade $46,20 \mathrm{~mm}$. Ingervall (1970) examinando 103 crianças na idade de 7 anos encontrou média de $46,40 \mathrm{~mm}$ (3). Agerberg (1974) registrou em 33 crianças com 1-2 anos de idade $38,40 \mathrm{~mm}$, e em 75 meninos e 75 meninas na idade de 6 anos $44,80 \mathrm{~mm}$ (1). Mais recentemente Kirveskari (1986) em 378 crianças com idades entre 5,10 e 15 anos de idade encontrou respectivamente a média de $40 \mathrm{~mm}$, $46 \mathrm{~mm}$ e $50 \mathrm{~mm}$.

Os resultados obtidos permitiram o estabelecimento de uma média variável com a faixa etária, e que, ao nosso ver, pode ser usada como base para estabelecimento de uma condição de normalidade que pode ser incluída na rotina do exame bucal em Odontopdiatria, como uma forma de detecção precoce de eventuais problemas no SNM ou da ATM. 


\section{CONCLUSÕES}

Face a análise dos resultados obtidos parece lícito concluir que:

1. Foram estabelecidos limites de normalidade de abertura bucal máxima para cada uma das faixas etárias avaliadas.

2. A técnica utilizada, pela sua simplicidade, pode ser incluída no exame clínico de rotina como auxiliar de diagnóstico.

3. Conforme os resultados obtidos qualquer valor, em qualquer faixa etária das analisadas, inferior a $36 \mathrm{~mm}$ deve levar o clínico a uma observação mais acurada, face a uma possivel disfunção do SEG.

\section{REFERÊNCIAS BIBLIOGRÁFICAS}

1. AGERBERG, G. Maximal mandibular movements in children. Acta Odont. Scand., 32:147-59, 1974.

2. ERIKSSON, E. The dependence of mandibular dysfunction in children on functional and morphologic malloclusion. Am. J. Or thod., 88(3):187-94, 1983.

3. INGERVALL, B. Range of movement of mandible in children. Scand. J. Dent. Res., 78:311-22, 1970.

4. LINDQVIST, B. Bruxism in twins. Acta Odontol. Scand., 32:177-87, 1974.

5. NEVAKARI, K. Elapsio prearticulris of the temporomandibular joint: a pantomographic study of the so called physiological subluxation. Acta Odontol. Scand., 18:123-70, 1960.
6. NILNER, M. Relationships between oral parafunctions and functional disturbances and diseases of the stomatognathic system among children aged 7-14 years. Acta Odontol. Scand., 411983.

7. NILNER, M. \& LASSING, S. Prevalence of functional disturbances and diseases of the stomatognathic system in 7-14 years old. Swed. Dent. J., 5:173-88, 1981.

8. PERSSON, M. et alii. Mandibular dysfunction in patients with rheumatoid arthritis. J. Craniomand. Disord. Facial Oral Pain, 2:201-08, 1988.

9. WOOLFOLK, M. Malocclusion and TMJ symptoms in migrant children. $J$. Craniomand. Disord. Facial Oral Pain, 2:196-200, 1988. 